\title{
Complete Thoracoscopic Segmentectomy and Pulmonary Lobectomy in the Treatment of Small Pulmonary Nodules
}

\begin{abstract}
Yu Zhou*
Department of Thoracic Surgery, Changshu Hospital Affiliated to Nanjing University of Chinese Medicine, Suzhou 215500, Jiangsu Province, China

*Corresponding author: Yu Zhou, introducewowo@sina.com

Abstract: Objective: To compare the clinical effect of complete thoracoscopic segmentectomy and pulmonary lobectomy for pulmonary nodules. Methods: In this study, 176 patients with pulmonary nodules were treated in Changshu Hospital Affiliated to Nanjing University of Chinese Medicine from January 2019 to June 2021; according to the type of surgery, the patients were divided into group A (complete thoracoscopic segmentectomy) and group B (complete thoracoscopic pulmonary lobectomy), and the clinical effects were analyzed. Results: The intraoperative blood loss, postoperative drainage volume, postoperative hospitalization days, and lung function of patients in group A were significantly better than those in group B $(P$ $<0.05$ ), while there was no significant difference in the number of dissected lymph nodes. Conclusion: The clinical effect of complete thoracoscopic segmentectomy for patients with small pulmonary nodules is more significant; it does not only ensure lymph node dissection, but also improve surgical-related indicators and treatment safety as well as speed up the recovery of pulmonary function. Its clinical application value is worthy of key analysis by medical institutions.
\end{abstract}

Keywords: Complete thoracoscopic segmentectomy; Pulmonary lobectomy; Small pulmonary nodules

Publication date: November 2021; Online publication: November 30, 2021

\section{Background}

Small pulmonary nodule refers to the existence of a single solitary pulmonary nodule with a diameter less than $3 \mathrm{~cm}$ in the lung. Its finding is not unusual during examinations. At present, most scholars believe that the main reason for the emergence of small pulmonary nodules is due to tumor, inflammation, congenital abnormalities, and other factors ${ }^{[1]}$. The vast majority of patients have no obvious symptoms; however, some lung cancers would manifest as solitary pulmonary nodules on chest radiographs, so it is necessary to pay more attention and carry out further examinations to determine whether surgical treatment is required ${ }^{[2]}$. Pulmonary lobectomy was commonly used in the past, but this kind of surgery leaves a negative impact on patients as they would have large surgical wounds, resulting in more postoperative complications; its overall curative effect also requires further improvement. With the development of medical technology, people have a deeper understanding of the lungs. Nowadays, segmentectomy is often used to preserve more lung tissue. In order to further compare the clinical effects of the two types of surgery, 176 patients with small pulmonary nodules were selected for this research.

\section{Materials and methods}

\subsection{General information}

A total of 176 patients with small pulmonary nodules, who underwent surgery in Changshu Hospital 
Affiliated to Nanjing University of Chinese Medicine from January 2019 to June 2021, were divided into two groups according to the type of surgery. Group A comprised of 89 patients who voluntarily underwent complete thoracoscopic segmentectomy; the average age of the patients was $56.75 \pm 12.98$ years old and the average nodule diameter was $1.11 \pm 0.33 \mathrm{~cm}$. Group B comprised of 87 patients who voluntarily underwent complete thoracoscopic pulmonary lobectomy; the average age of the patients was $59.91 \pm 11.11$ years old and the average nodule diameter was $1.23 \pm 0.47 \mathrm{~cm}$. The baseline data of the two groups were compared and found to be comparable $(P>0.05)$.

\subsection{Method}

Each patient was placed in a lateral position and underwent general anesthesia with endotracheal intubation as well as single lung ventilation. An incision of about $1 \mathrm{~cm}$ was made in the 7 th rib at the midaxillary line. Under thoracoscopic guidance, an incision of about $4 \mathrm{~cm}$ was made between the anterior axillary line and the midclavicular line between the 3rd and 4th ribs as the main opening, while an incision of about $2 \mathrm{~cm}$ was made between the 7th and 8th ribs at the midaxillary line as the secondary opening. After blunt separation, thoracoscopy was used to remove the lung lesions; the size and shape of the pulmonary nodules were observed, and a rapid pathological examination was conducted. Based on the results, 89 patients underwent segmentectomy and 87 patients underwent pulmonary lobectomy with routine postoperative treatment.

\subsection{Observation indexes}

The surgical indicators of the two groups were observed and compared, including lymph node dissection, intraoperative blood loss, postoperative drainage, and postoperative hospital stay. The lung function of the patients in the two groups before treatment and 3 months after treatment were also compared. The patients' forced expiratory volume/predicted value $\left(\mathrm{FEV}_{1}\right)$, maximum ventilatory volume per minute/predicted value (MVV), and forced vital capacity/predicted value (FVC) in one second were measured using a pulmonary function instrument and were statistically compared.

\subsection{Statistical analysis}

R 3.5.3 software was used to analyze the collected data. The measurement data were expressed in $\bar{x} \pm \mathrm{s}$, and t-test was applied. $P<0.05$ was considered to be statistically significant.

\section{Results}

\subsection{Surgical indicators}

There was no significant difference in the number of intraoperative lymph node dissection between the two groups $(P>0.05)$. The intraoperative blood loss, postoperative drainage volume, and postoperative hospital stay of group A were $58.61 \pm 16.96 \mathrm{ml}, 500.53 \pm 287.22 \mathrm{ml}$, and $5.74 \pm 1.78$ days, respectively, which were significantly better than those of group B $(P<0.05)$ (Table 1). 
Table 1. Comparison of surgical indicators between the two groups of patients with pulmonary nodules $(\bar{x}$ $\pm s)$

\begin{tabular}{cccccc}
\hline Group & $\begin{array}{c}\text { Number } \\
\text { of cases }\end{array}$ & $\begin{array}{c}\text { Number of lymph } \\
\text { node dissection }\end{array}$ & $\begin{array}{c}\text { Intraoperative } \\
\text { blood loss }(\mathrm{ml})\end{array}$ & $\begin{array}{c}\text { Postoperative } \\
\text { drainage }(\mathrm{ml})\end{array}$ & $\begin{array}{c}\text { Postoperative } \\
\text { hospital stay (days) }\end{array}$ \\
\hline Group A & 89 & $8.86 \pm 2.67$ & $58.61 \pm 16.96$ & $500.53 \pm 287.22$ & $5.74 \pm 1.78$ \\
Group B & 87 & $9.68 \pm 4.35$ & $67.27 \pm 22.21$ & $859.90 \pm 637.58$ & $7.14 \pm 2.97$ \\
$\mathrm{t}$ value & - & 1.517 & 2.909 & 4.839 & 3.812 \\
$P$ value & - & 0.133 & 0.004 & 0.000 & 0.000 \\
\hline
\end{tabular}

\subsection{Lung function indicators}

The FEV $1(84.30 \pm 5.18 \%)$, MVV $(83.73 \pm 5.26 \%)$, and FVC $(85.23 \pm 5.07 \%)$ of group A were significantly better than those of group B $(P<0.05)$ (Table 2).

Table 2. Comparison of lung function indicators between the two groups of patients with pulmonary nodules $(\bar{x} \pm s)$

\begin{tabular}{ccccc}
\hline Group & Number of cases & $\mathrm{FEV}_{1}(\%)$ & MVV $(\%)$ & FVC $(\%)$ \\
\hline Group A & 89 & $84.30 \pm 5.18$ & $83.73 \pm 5.26$ & $85.23 \pm 5.07$ \\
Group B & 87 & $75.19 \pm 5.55$ & $76.54 \pm 5.32$ & $77.67 \pm 4.85$ \\
t value & - & 11.244 & 9.009 & 10.092 \\
$P$ value & - & 0.000 & 0.000 & 0.000 \\
\hline
\end{tabular}

\section{Discussion}

In most cases, small pulmonary nodules grow insidiously in the lungs like "lurkers," as they do not cause obvious discomfort symptoms to the patients. They can only be seen on chest radiographs when their diameter exceeds $1 \mathrm{~cm}^{[3]}$. The clinical treatment is based on the nature of the small nodules, whether they are benign or malignant. Benign nodules can be observed closely, whereas malignant pulmonary nodules need to be surgically removed as soon as possible, so as to avoid complications, such as pneumonia, atelectasis, obstructive diseases, and so on ${ }^{[4]}$. The surgical resection of lung lobes is often the choice to achieve the purpose of radical cure; however, it has a negative impact on patients' lung function after the surgery. A long recovery time is required by these patients, and the incidence of complications increases, thus reducing the curative effect of patients. Segmentectomy was initially used in the treatment of bronchiectasis. Based on human anatomy, the left lung can be subdivided into eight lung segments, while the right lung can be subdivided into ten lung segments. Through segmentectomy, lung tissues can be retained as much as possible to reduce the impact of the surgery on the patient ${ }^{[5]}$. With the help of thoracoscopy, it does not only significantly reduce the surgical wound, but also provide doctors with sufficient vision to observe the patient's lung condition, and conduct rapid pathological examination after lesion resection, so as to determine whether the patient is suitable for thoracoscopic segmentectomy ${ }^{[6]}$. For patients whose results show that the cutting edge or lymph nodes are positive, lobectomy must be performed to remove the focus and lymph nodes as much as possible, in order to reduce the possibility of cancer cell proliferation, ensure the prognosis of patients, and reduce the probability of disease recurrence ${ }^{[7]}$. For patients with negative examination results, only segmental pneumonectomy is required; this surgery improves the surgical indicators and accelerates the recovery process. 
The results showed that there was no significant difference in the number of intraoperative lymph node dissection between the two groups $(P>0.05)$; the amount of intraoperative bleeding, postoperative drainage, and postoperative hospital stay of patients in group A were significantly better than those of group B $(P<$ 0.05); at the same time, the $\mathrm{FEV}_{1}, \mathrm{MVV}$, and FVC of group A were significantly better than those of group B $(P<0.05)$, suggesting that complete thoracoscopic segmentectomy can reduce the damage to patients' body and shorten their hospital stay on the premise of ensuring the curative effect, which has a positive significance for improving patients' lung function and prognosis, as well as ensuring the treatment safety and improving the long-term curative effect.

In conclusion, for patients with small pulmonary nodules whose rapid pathological examination results reveal that the cutting edge or lymph nodes are positive, pulmonary lobectomy should be the choice of treatment to remove as many cancer cells as possible, ensure short-term and long-term curative effects, as well as avoid further adverse effects on the body caused by the recurrence of the disease. However, if the pathological examination results are negative, complete thoracoscopic segmentectomy provides better overall curative effect, effectively reduces the negative impact of surgery on patients' lung function, improves the safety of surgery, and enables patients to return to their normal life as soon as possible. It also has high applicability for patients with physical deficiency and of old age; thus, it is worthy of clinical promotion.

\section{Disclosure statement}

The author declares that there is no conflict of interest.

\section{References}

[1] Yu F, He J, 2019, Recent Efficacy Comparison of Complete Thoracoscopic Anatomic Segmentectomy and Pulmonary Lobectomy in the Treatment of Small Pulmonary Nodules. World Latest Medicine Information (Continuous Electronic Journal), 19(95): 25-26.

[2] Yu G, Huang B, Mi Y, et al., 2019, Short and Long Term Efficacy and Safety of Complete Thoracoscopic Anatomic Segmentectomy in the Treatment of Small Pulmonary Nodules. Guangdong Medical Journal, 40(13): 1872-1875.

[3] Tang H, Mei H, Xu C, et al., 2017, Clinical Observation of Complete Thoracoscopic Anatomic Segmentectomy in the Treatment of Small Pulmonary Nodules. Hainan Medical Journal, 28(3): 411413.

[4] Zhang L, Xue R, Wu X, 2018, Feasibility of Complete Thoracoscopic Anatomic Segmentectomy in the Treatment of Small Pulmonary Nodules. China Health Care \& Nutrition, 28(4): 17.

[5] Pang W, Huang F, Ye M, et al., 2017, Prospective Study of Complete Thoracoscopic Anatomic Segmentectomy Versus Pulmonary Lobectomy in the Treatment of Early Lung Cancer. Journal of Clinical Pulmonary Medicine, 22(10): 1874-1878, 1883.

[6] Zhang R, Shi C, 2014, Clinical Analysis of Complete Thoracoscopic Segmentectomy and Pulmonary Lobectomy in the Treatment of Small Pulmonary Nodules. Contemporary Medicine, (27): 83-84.

[7] Song X, Ren M, Meng Q, et al., 2014, Comparison of Short-Term Effect of Thoracoscopic Segmentectomy and Thoracoscopic Lobectomy for the Solitary Pulmonary Nodule and Early-Stage Lung Cancer. Onco Targets Ther, 2014: 1343-1347. 